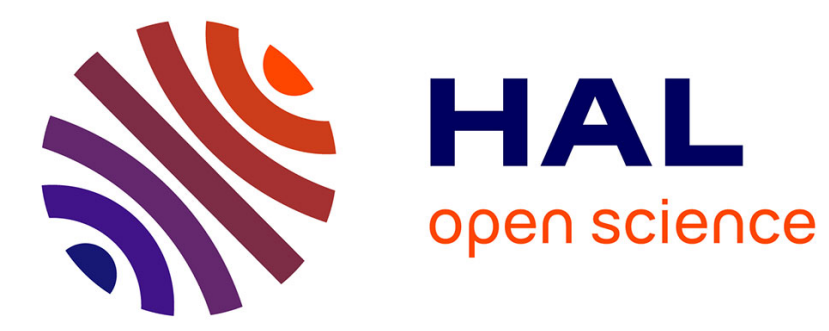

\title{
Les fondations mythiques de Sanaa et d'Aden
}

Franck Mermier

\section{To cite this version:}

Franck Mermier. Les fondations mythiques de Sanaa et d'Aden . Revue du monde musulman et de la méditerranée, 1993. halshs-01855319

\section{HAL Id: halshs-01855319 https://shs.hal.science/halshs-01855319}

Submitted on 7 Aug 2018

HAL is a multi-disciplinary open access archive for the deposit and dissemination of scientific research documents, whether they are published or not. The documents may come from teaching and research institutions in France or abroad, or from public or private research centers.
L'archive ouverte pluridisciplinaire HAL, est destinée au dépôt et à la diffusion de documents scientifiques de niveau recherche, publiés ou non, émanant des établissements d'enseignement et de recherche français ou étrangers, des laboratoires publics ou privés. 


\title{
Persée
}

\author{
http://www.persee.fr
}

\section{Les fondations mythiques de Sanaa et d'Aden}

\section{Franck Mermier}

Mermier Franck, . Les fondations mythiques de Sanaa et d'Aden. In: Revue du monde musulman et de la Méditerranée, №67, 1993. Yémen, passé et présent de l'unité. pp. 131-139.

Voir l'article en ligne

\section{Avertissement}

L'éditeur du site «PERSEE » - le Ministère de la jeunesse, de l'éducation nationale et de la recherche, Direction de l'enseignement supérieur, Sous-direction des bibliothèques et de la documentation - détient la propriété intellectuelle et les droits d'exploitation. A ce titre il est titulaire des droits d'auteur et du droit sui generis du producteur de bases de données sur ce site conformément à la loi n` $98-536$ du $1 \mathrm{er} \mathrm{juillet} 1998$ relative aux bases de données.

Les oeuvres reproduites sur le site «PERSEE » sont protégées par les dispositions générales du Code de la propriété intellectuelle.

Droits et devoirs des utilisateurs

Pour un usage strictement privé, la simple reproduction du contenu de ce site est libre.

Pour un usage scientifique ou pédagogique, à des fins de recherches, d'enseignement ou de communication excluant toute exploitation commerciale, la reproduction et la communication au public du contenu de ce site sont autorisées, sous réserve que celles-ci servent d'illustration, ne soient pas substantielles et ne soient pas expressément limitées (plans ou photographies). La mention Le Ministère de la jeunesse, de l'éducation nationale et de la recherche, Direction de l'enseignement supérieur, Sous-direction des bibliothèques et de la documentation sur chaque reproduction tirée du site est obligatoire ainsi que le nom de la revue et- lorsqu'ils sont indiqués - le nom de l'auteur et la référence du document reproduit.

Toute autre reproduction ou communication au public, intégrale ou substantielle du contenu de ce site, par quelque procédé que ce soit, de l'éditeur original de l'oeuvre, de l'auteur et de ses ayants droit.

La reproduction et l'exploitation des photographies et des plans, y compris à des fins commerciales, doivent être autorisés par l'éditeur du site, Le Ministère de la jeunesse, de l'éducation nationale et de la recherche, Direction de l'enseignement supérieur, Sous-direction des bibliothèques et de la documentation (voir http://www.sup.adc.education.fr/bib/ ). La source et les crédits devront toujours être mentionnés. 


\section{Franck Mermier}

\section{LES FONDATIONS MYTHIQUES DE SANAA ET D'ADEN}

Depuis la proclamation de l'Unité, le 22 mai 1990, Sanaa est devenue la capitale du Yémen et Aden sa capitale économique. La réunification du pays a drainé vers Sanaa la plupart des dirigeants politiques de l'ex-Yémen du Sud qui partagent aujourd'hui le pouvoir avec leurs anciens adversaires idéologiques du Nord. La capitale des hauts-plateaux zaydites que se disputèrent, dans l'histoire récente, les imams et les walis ottomans (1872-1918) puis les royalistes et les républicains (1962-1970), est sortie de ses murs pour accueillir toutes les communautés du pays, formant ainsi une société hybride et composite mais tournée, pour une large part, vers ses origines multiples et ses allégeances communautaires. L'exode rural et l'attraction des élites se sont en effet conjugués pour stimuler de manière décisive la croissance urbaine. Pourtant, ce caractère mosaïque de la ville, qui n'a pas encore donné lieu à une véritable culture urbaine, se trouve confronté au défi d'un environnement tribal qui peut imposer ses règles au cœur même de la ville sinon au sein même de l'Etat. Depuis les hauts-plateaux où prédomine le système tribal jusqu'aux rivages de l'océan Indien, l'Unité a été proclamée, la circulation est de nouveau possible mais par là même, de nouvelles injustices ont essaimé. Aden, déchirée par la guerre civile sanglante de 1986, aurait pu connaître quelque répit après la fin d'un régime totalitaire brusquement converti à la démocratie. Cela n'a pas été le cas, la ville n'est certes pas devenue, comme Sanaa, le théâtre épisodique de règlements de comptes entre tribus mais elle s'est trouvée insidieusement asphyxiée par la centralisation des ressources étatiques dans la capitale et insécurisée par les prédateurs de la libéralisation mercantiliste. Son poumon économique, comme au temps de l'occupation britannique (1839-1967), n'est plus la mer, quasimentdéserte, mais la route qui la relie à la capitale et au véritable port du pays, Hodeydah, où se déversent la majeure partie des biens de consommation. Autrefois phare de la révolution marxiste dans le monde arabe, Aden, repliée au fond de sa baie, est aujourd'hui subordonnée à Sanaa et marginalisée sur le plan économique malgré son statut théorique de deuxième ville du pays.

Reflétant leur statut privilégié dans l'histoire du Yémen, ces deux villes occupent une place particulière dans la constellation de son répertoire mythique. De fait, leurs récits de fondation qui se 


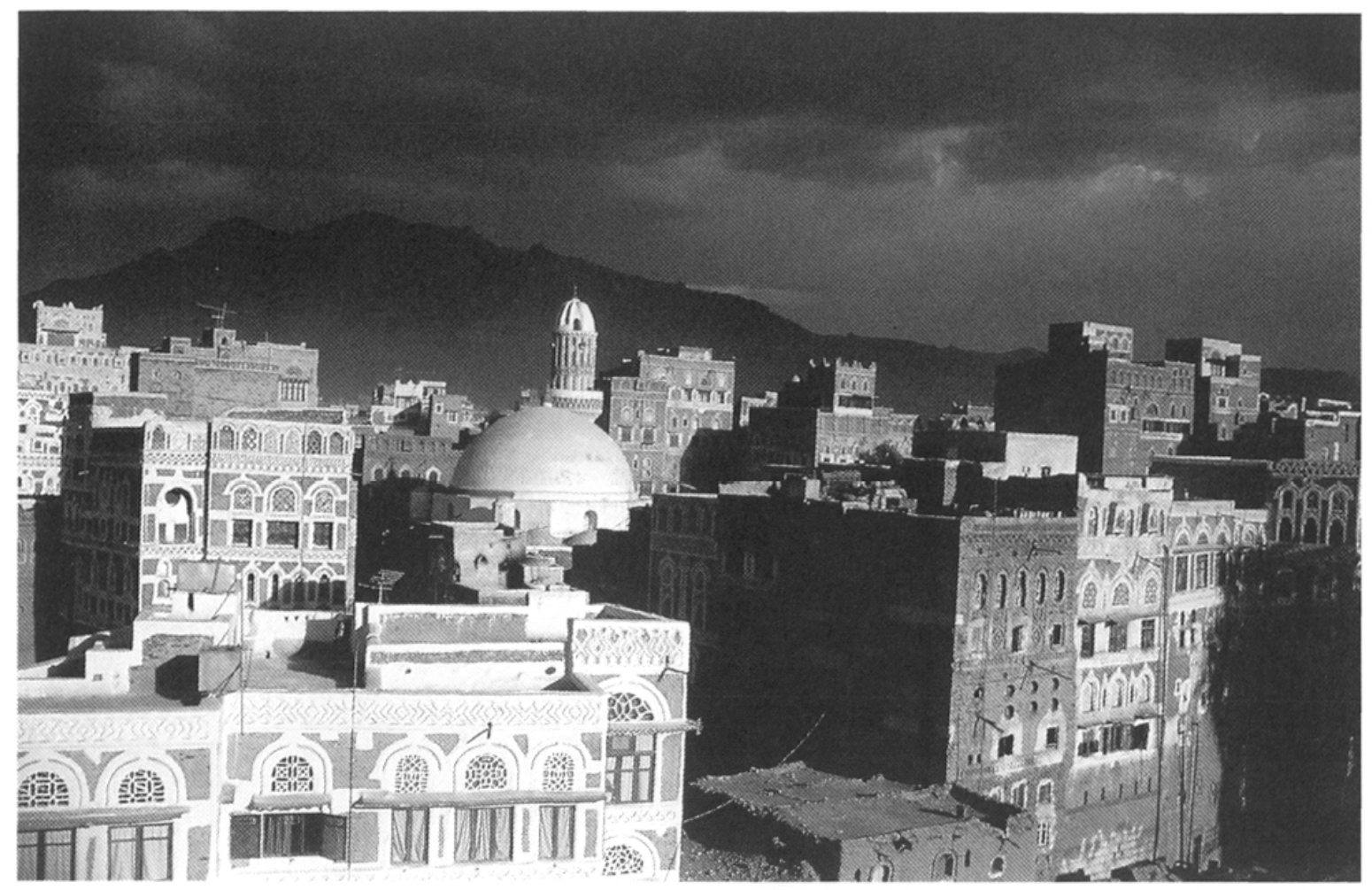

Sanaa avec au fond le djebel Nuqum dominant la ville

particularisent par un caractère épique plus marqué ont laissé une trace plus importante dans les sources arabes que ceux d'autres villes du Yémen. A l'instar d'autres cités islamiques, les origines mythiques d'Aden et de Sanaa les rattachent à une géographie sacrée dont les centres sont, par ordre de sacralité, La Mecque, Médine et Jérusalem'. Il convient cependant de préciser que ces récits étaient tenus pour historiques et qu'aucune distinction n'était établie entre la part d'histoire avérée et la part de légendes, de même qu'entre temps mythique et temps historique. C'est bien notre "regard éloigné" nourri de sa propre vision de l'histoire qui permet, ou se permet, d'en isoler les éléments mythiques². L'actualité de ces mythes est aujourd'hui bien faible ; ils sont délaissés par la transmission orale et restent enclos dans des ouvrages, ceux que nous avons consultés ayant été écrits entre le $\mathrm{X}^{\mathrm{e}}$ et le $\mathrm{XVI}^{\mathrm{e}}$ siècles, constituant aujourd'hui une strate de l'imaginaire rarement réactivée ${ }^{3}$. Pourtant, ces récits de fondation nous semblent devoir susciter plus qu'un simple intérêt anecdotique du fait qu'ils révèlent un ensemble de représentations symboliques en partie liées à la ville mais la prenant, bien souvent, pour prétexte. On pourrait même se demander si, autour d'Aden et de Sanaa, ce n'est pas une partie de "l'épopée qahtanide" (M. Piotrovsky, 1984 et 1986) qui se cristallise pour rencontrer l'épopée coranique. Il s'avère en effet que, dans le temps du mythe, un lien profond existe entre Aden et Sanaa : leur position éminente dans le cadre d'une métahistoire musulmane réinterprétée au Yémen et élaborée dans le souci de rattacher l'Arabie du Sud au cycle prophétique d'avant la Révélation. Leurs origines mythiques sont ainsi liées aux pérégrinations de héros coraniques, bénéfiques ou maléfiques, qui parcourent la terre en quête du lieu le plus propice pour y fonder une ville. L'élaboration mythique, qu'il s'agisse de la fondation de Sanaa ou d'Aden, puise dans les mêmes catégories de pensée et manifeste une unicité de l'imaginaire qui sous-tend un système de relations subtiles entre les structures narratives de ces récits de fondation. Partant de ces réflexions, il nous a semblé pertinent d'esquisser une brève comparaison entre les origines légendaires de ces deux villes pour tenter de poser quelques jalons d'analyse et rappeler que l'unité du Yémen peut aussi être captée dans l'ordre de l'imaginaire. 


\title{
Sanaa ou l'épure d'une fondation
}

L'origine mythique de Sanaa est directement liée à la fondation du palais de Gumdān. Celui-ci est, selon la légende, le premier édifice à avoir été construit par Sem après qu'il ait choisi le site pour fonder la première ville après le déluge. Le savant yéménite, al-Hamdāni (décédé dans la deuxième moitié du $\mathrm{X}^{\mathrm{e}}$ siècle) est un des premiers à en faire le récit :

\begin{abstract}
"Sem, fils de Noé, a fondé Gumdān. Il a creusé son puits qui sert, aujourd'hui, de réservoir à la Grande mosquée de Sanaa. Ayant parcouru les terres du nord, il se rendit dans le sud, visitant les pays les plus agréables, et arriva dans le premier "climat". Là, le Yémen lui apparut comme l'endroit le plus propice pour y élire domicile. Après un si long voyage, il arriva dans la plaine de Sanaa. Il posa son cordeau entre les deux montagnes surplombant Gumdān à l'ouest de la plaine de Sanaa et construisit "al-zibr" qui existe encore aujourd'hui. Lors de sa construction, Dieu envoya un oiseau qui enleva le cordeau. Sem le suivit pour voir où il tomberait. Il s'arrêta au sud du Na'ìm au pied de la montagne Nuqum et le lâcha mais quand Sem l'atteignit, l'oiseau le reprit et le posa sur les tells volcaniques de Gumdān. Lorsque le cordeau fut fixé sur la plaine de Gumdān, Sem comprit qu'il lui avait donné l'ordre de construire en ce lieu. Il fonda donc Gumdān puis creusa son puits appelé "karāma" et utilisé encore de nos jours bien que son eau soit saumâtre ${ }^{4}$."
\end{abstract}

Ibn al-Dayba“ (1992 : 130-131) propose une autre variante moins riche en détails mais apportant, en liaison avec Sanaa, des données inédites sur la progéniture de Sem. Sur ordre de son père Noé, celui-ci se dirigea avec ses enfants en direction du Yémen à la recherche d'un endroit où élire sa demeure. Négligeant le Hédjaz, le Nejd et la région d'al-'Arụ̣̄ dont les climats ne lui convenaient pas, il s'arrêta à Sanaa pour y fonder la ville et y creuser un puits qui porte son nom. Pendant ce temps, son frère Yāfit (Japhet) s'établissait dans les terres du nord et Hāām (Chem), ancêtre des Africains, dans les régions sud de la terre. A Sanaa, Sem eut cinq enfants, Arfaȟšad, Ašūd̄, Lāwad̄, Iram et 'Awilam qui, sous l'influence du climat, virent leur langage et leurs aspects physiques se transformer. Ils acquirent ainsi le type ethnique des Arabes et en devinrent les ancêtres.

Le géographe Ibn al-Muğāwir (1986 : 179-183) nous rapporte des versions quelque peu différentes de ce mythe en se fondant sur l'autorité d'auteurs anciens ${ }^{5}$. Selon l'un d'eux, ce serait Seth, un des fils d'Adam qui aurait construit Sanaa et aurait planté, à sa périphérie, deux jardins placés des deux côtés d'une route et dont la longueur équivalait à sept journées de marche entre Sanaa et l'Irak. Un autre rapporte sa fondation à l'œuvre de Sem qui, à la recherche d'un lieu pouvant alléger sa douleur et pourvu d'un climat tempéré, d'eau douce et d'une terre bénéfique, découvrit que Sanaa était l'endroit le plus propice. Il monta sur la montagne Nuqum et ordonna à ses gens de se construire chacun une maison. La ville atteignit bientôt une largeur et une longueur de sept parasanges ${ }^{6}$ et ses dépendances s'étendirent jusqu'à Bașra. Une route reliait ces deux cités; elle resta pratiquable "jusqu'à ce qu'elle fut recouverte par les sables". Le premier puits de la Création y fut creusé par Hūd, un prophète pré-islamique dont le corps reposerait, dit-on, dans le sanctuaire qui lui est consacré près de Țarīm (Hadramawt). Le palais de Gumdān est lui-aussi mentionné comme ayant été fondé par Sem puis rehaussé d'une demeure royale par chaque tubba 'yéménite jusqu'à atteindre " 72 ou 93 niveaux" . Le dernier à l'avoir surélevé fut As'ad al-Kāmil, un des tubba ' les plus célèbres dont les exploits légendaires sont souvent empruntés à l'épopée d'Alexandre le Grand et qui, dans les anciennes légendes yéménites, est identifié au tubba coranique (M. Piotrovsky, 1984 : 104-108 et 74) ${ }^{8}$. Dans la version d'al-Hamdānī, un oiseau manifeste la toute puissance de Dieu en guidant l'acte fondateur. Ce thème n'est pas propre à Sanaa, il se retrouve dans d'autres récits de fondation de villes musulmanes, Le Caire et Alexandrie notamment ${ }^{9}$. Dans le cas de Sanaa, la volonté de valoriser la civilisation des Arabes du sud, les Qahtạn, est sous-jacente à son origine mythique qui allie l'âge d'or pré-islamique, Gumdān et As'ad al-Kāmil, et une généalogie sacrée, Sem, Seth et Hūd. Elle pénètre aussi le thème de la protection divine de Sanaa que plusieurs légendes viennent illustrer. Al-Hamdãni (1986:22) rapporte que ses habitants, lors d'un combat avec leurs ennemis, entendirent une voix surnaturelle les assurer de la miséricorde de Dieu pour 


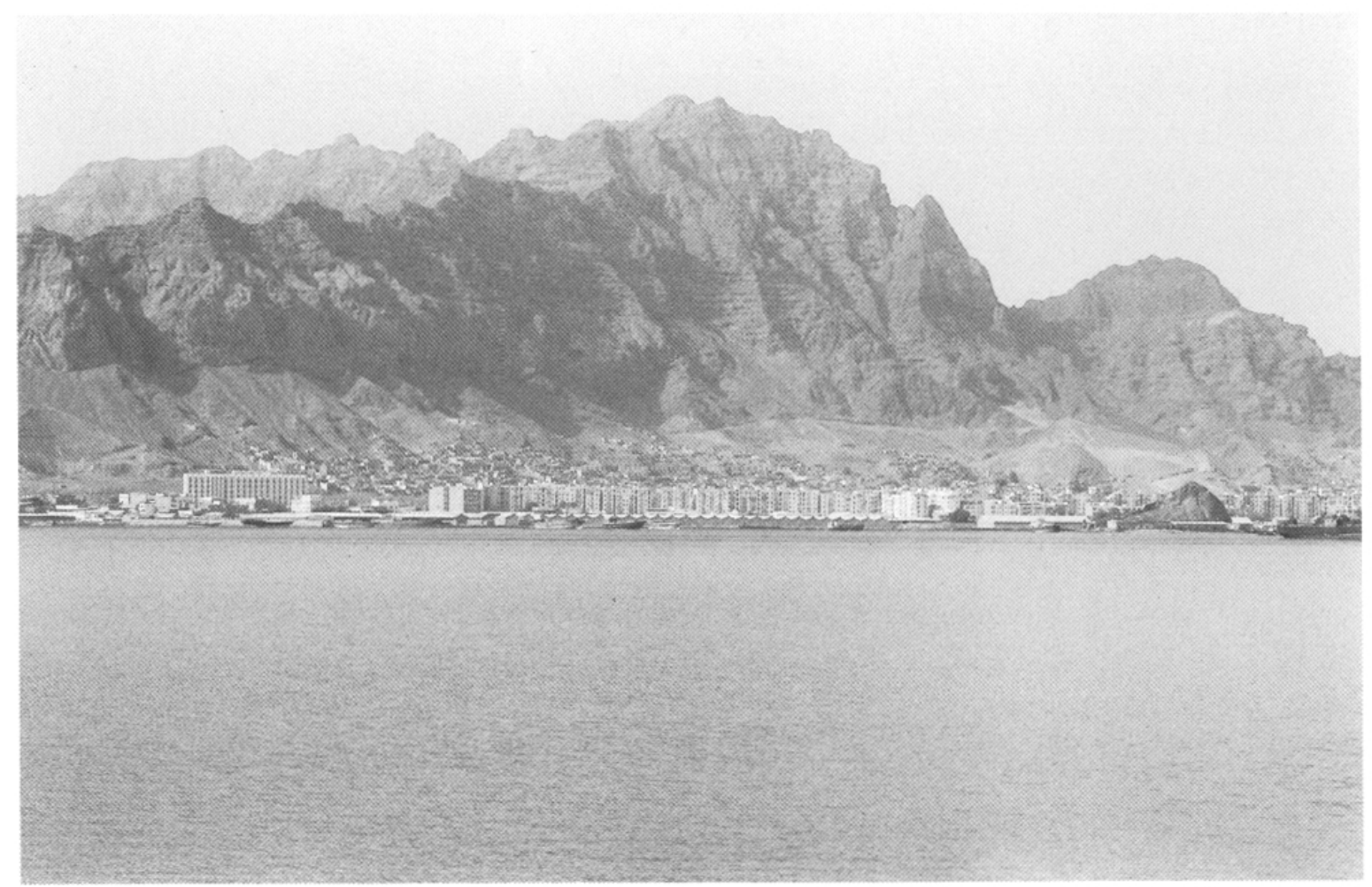

Aden vu de la mer (photo Jean-Charles Depaule).

Azāl, le nom légendaire du Sanaa anté-islamique. C'est le chant d'un oiseau, répétant "le village est protégé", que Wahb b. Munabbih entendait chaque jour à l'extérieur de la ville. Le témoignage de ce personnage est invoqué par al-Rāzì (1986 : 33-36), un auteur yéménite du XI ${ }^{\mathrm{e}}$ siècle, pour relater comment une gazelle provoqua le massacre des assiégeants de Sanaa, des hommes de tribu de Hamdān, qui s'entretuèrent pour sa possession. L'armée d'un gouverneur omeyyade campant aux portes de la cité connut le même sort, prenant pour une attaque nocturne l'arrivée de montures échappées de la ville. Les serpents même ne pouvaient pénétrer en ville :

"Sanaa est protégée par deux talismans représentant des serpents et peu s'en faut qu'un habitant de cette ville en ait été victime. De mémoire d'homme, aucun de ceux qui en ont été mordus n'en est mort et cette victoire reste unique. Posés sur les portes de la ville, un de ces talismans était en fer et l'autre en cuivre..." (al-Rāzì, 1986 : 203-204).

Tel un microcosme réfléchissant par réfraction la mythologie coranique ou le temps mythique de la complétude religieuse, l'enceinte de la ville devient le théâtre d'une histoire sainte enracinant le lieu dans un temps primordial où se succédaient les envoyés de Dieu. Ibn Rustah rapporte ainsi une tradition qui accrédite la volonté de sacralisation de la ville par ses habitants :

"Une grande arcade en pierre se dresse près du marché des bouchers : ce fut là, prétend la population, que furent égorgés autrefois seize prophètes” (Ibn Rustah,1955 : 125).

\section{Aden, entre terre et mer}

Dans le récit de fondation d'Aden que nous rapporte Abū Muḥammad 'Abd Allāh ... Abū Mahrama (1991), décédé en 947/1540, le site est visité aux temps anciens par Qābil (Caïn), fils d'Adam, et Dū al-Qarnayn, le Bi-Cornu mentionné dans le Coran ${ }^{10}$. Le premier est évidemment un personnage maléfique alors que le second est considéré comme un prophète par certains exégètes musulmans qui l'assimilent à un tubba' yéménite (M. Gaudefroy-Demonbynes, 1969 : 370). Dans 
ce récit, Qābil ne joue aucun rôle dans la fondation de la ville; s’il élit domicile sur le ğabal Sïra qui surplombe Aden, c'est parce qu'il est venu s'y réfugier après le meurtre de son frère :

"Qābil, fils d'Adam et meurtrier de son frère Abel, s'enfuit de l'Inde par crainte de son père et arriva à Aden avec sa famille. Il séjourna sur le ğabal Sira mais bientôt le mal du pays s'empara de lui et alors Iblis lui apparût, ayant apporté des flûtes et en jouant pour le distraire" (Abū Mahrama, $1991: 7-8)$.

Un parfum d'ambivalence imprègne donc ce récit, le voyage de Qābil à Aden est placé en introduction de la visite de Dū al-Qarnayn, mais sans aucune transition comme une pièce rapportée venue d'un autre corps de légendes. Du fait de la présence du meurtrier d'Abel, le site, malgré la proximité temporelle du paradis perdu, ne suggère en rien l'idée d'un Eden. Pourtant selon Yãqūt, la ville légendaire d'Iram aux piliers aurait été construite près d'Aden, à l'image du Paradis, par Šaddād b. 'Ād. Mais celui-ci fut châtié de son orgueil et sa ville détruite par une intervention divine"'. Dans la version rapportée par Ibn al-Muğāwir, la mention de Qābil ne figure pas ; l'histoire commence avec Dũ al-Qarnayn et la fondation de la ville est le fait de Šaddād b. 'Ād. Pour le reste, elle est sensiblement identique à celle d'Abū Mahrama. C'est la version d'Ibn al-Muğāwir (1986 : 106-108) que nous reproduisons ici :

\begin{abstract}
"Depuis al-Qulzum jusqu'à Aden et en-deçà de la montagne de Socotra, existait une seule bande de terre. Dū al-Qarnayn, au cours de ses pérégrinations à travers le monde, arriva en ce lieu et ouvrit un canal dans la mer qui s'y engouffra jusqu'à la montagne de Bāb al-Mandab. Aden fut ainsi entourée par les eaux et seuls étaient visibles les sommets des montagnes qui ressemblaient à des îles. Une claire indication de ce fait est qu'une nappe d'eau se trouve encore sur les sommets du ğabal al-'Urr, de la montagne sur laquelle fut édifiée la citadelle d'al-Ta'kar et du ğabal al-Ahdar. Une autre est que Šaddād ibn 'Ād ne construisit Iram aux piliers (Iram ḍāt al-'imād), entre al-Laḩaba, Laḥ̆g et alMagāāwi située sur la route d'al-Mafāliss, cette bande de sable conduisant au ğabal Dār Zĩna, que sur la meilleure et la plus pure des terres, éloignée de la mer, et dans une atmosphère des plus propices. Maintenant, la mer a recouvert les extrémités du pays d'Irām aux piliers et en a submergé une partie lorsque Dū al-Qarnayn perça une ouverture depuis l'île de Socotra et que les flots atteignirent les confins d'al-Mandab. La troisième indication est que la mer située entre al-Sirrayn et Djedda s'appelle Matạarid al-hayl et Marābiṭ al-ḩayl soit que les Arabes attachaient leurs chevaux à cet endroit soit qu'ils les chassaient sur une terre ferme antérieure à la mer... Après que Dū al-Qarnayn eut ouvert le Bāb al-Mandab, la mer s'y engouffra jusqu'à atteindre l'extrêmité d'al-Qulzum et la terre d'Aden apparût. Abū 'Abd Allāh Muhammad bin 'Abd Allāh al-Kìsānī mentionne dans son commentaire coranique (tafsir) que Šaddād, le fils de 'Ād, lors de son départ du Yémen pour le Hadramawt, arriva à Lahğ et vit de très loin le ğabal al-'Urr. Il dit alors à ses soldats, venez et regardez cette montagne et ce qui se trouve en-deçà. En voyant cet endroit, ils s'en retournèrent et dirent que ce lieu était une vallée et qu'en son milieu se trouvait un arbre dominant la mer et habité par d'énormes serpents. Lorsque Šaddād entendit ce propos, il partit à Lahğ et ordonna de creuser les puits qui abreuvent aujourd'hui les habitants d'Aden et de percer une porte dans la partie la plus élevée de la vallée ${ }^{12}$."
\end{abstract}

Ibn al-Muğāwir poursuit son récit en précisant que deux génies à forme humaine, selon les sages indiens, furent chargés, l'un de percer la porte, l'autre de creuser une rivière depuis le sommet de Socotra jusqu'à Aden. Le premier acheva sa tâche après un labeur de soixante dix ans, le second ne put jamais la terminer et s'arrêta au pied du ğabal Ḥadid (la montagne de fer). Šaddād choisit l'emplacement de la porte pour en faire une prison qui fut utilisée "jusqu'à la fin de l'empire des Pharaons".

\title{
Regards croisés
}

La lecture des récits de fondation de Sanaa et d'Aden fait ressortir des différences notables dans l'élaboration mythique relative à ces deux cités. En comparant le statut respectif des personnages qui, dans la partition imaginaire de leur origine, jouent un rôle éminent, il apparaît que Sanaa occu- 


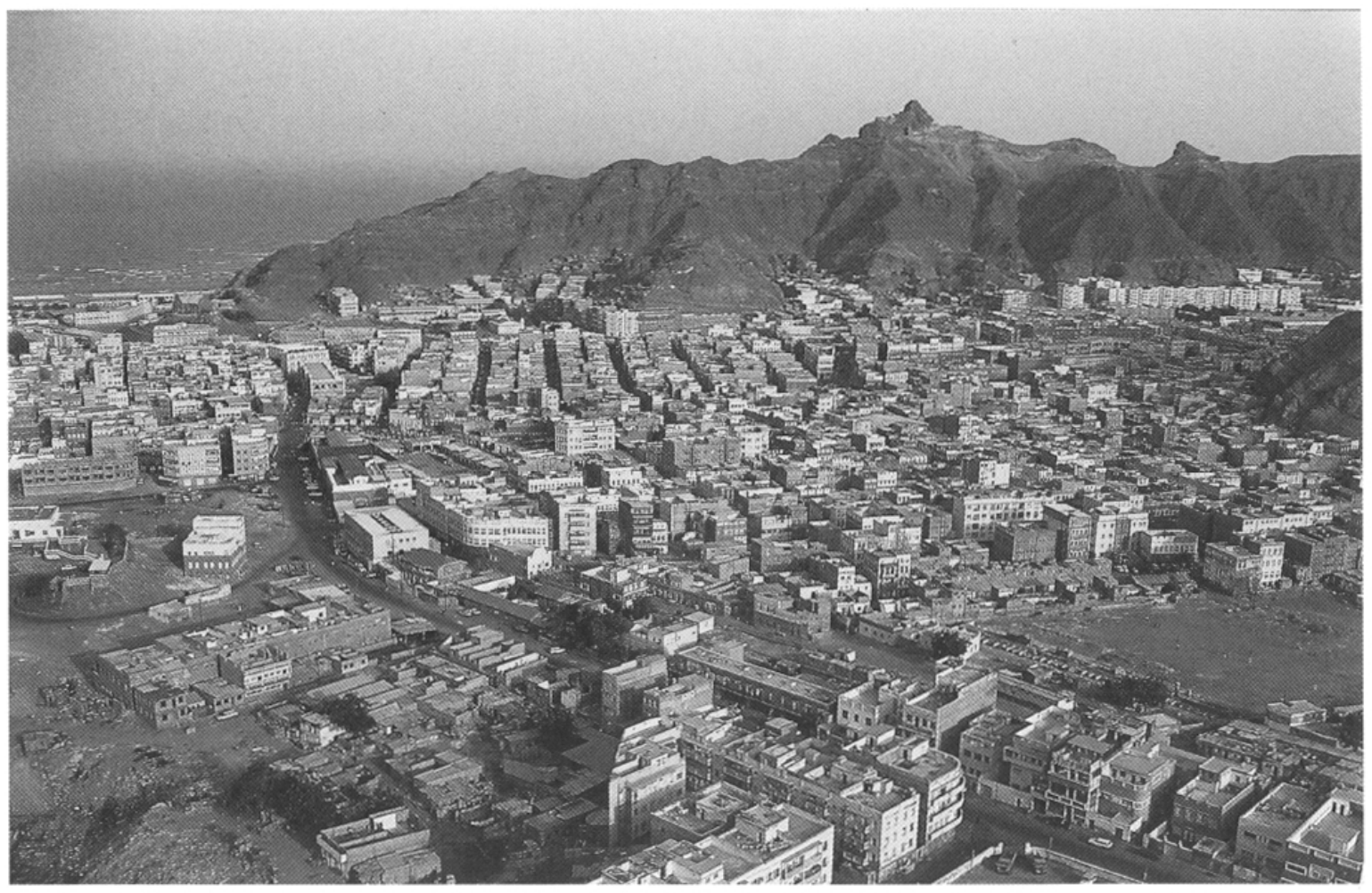

Aden nichée au fond d'un cratère volcanique qu'une échancrure ouvre sur l'océan. Contrairement à Sanaa, les bâtiments de la ville sont presque tous modernes. En effet lorsque les Anglais s'y installèrent en 1839, la ville était largement en ruine et ne comptait guère plus d'un millier d'habitants.

pe un rang nettement supérieur à celui d'Aden. Sem, Seth, Hūd et As'ad al-Kāmil apposent sur cette première cité leur aura bénéfique alors que Qābīl et Šaddād, héros négatifs, marquent le site d'Aden d'un stigmate maléfique. En un certain sens, cette opposition prolonge le conflit entre le Prophète Hūd et le peuple de 'Ād qui a rejeté son message et dont l'orgueil s'est incarné dans Šaddād et sa volonté de créer un paradis terrestre. Mais si l'on choisit de ne pas exagérer l'importance de Hūd dans la fondation de Sanaa respectant en cela la rareté de ses occurences, il est par contre aisé de retrouver un autre couple de contraires. Šaddād est en effet considéré comme un descendant de Noé et, du fait de son refus de la Révélation divine transmise par Hūd, s'oppose donc à Sem comme Qābīl à Abel. Dans une certaine mesure, l'acte fondateur de Dū al-Qarnayn vient atténuer les effets pernicieux de ce patronage encombrant. Mais celui-ci ne fonde pas la cité, il redessine les frontières de l'espace terrestre et de l'élément marin comme si, dans ce récit, il reproduisait le mur qu'il avait édifié pour contenir les peuples maudits de Gog et Magog ${ }^{13}$. Un parallèle peut être tracé entre le rôle de Dū al-Qarnayn à Aden et celui d'As'ad al-Kāmil à Sanaa si l'on se réfère aux poèmes de l'épopée qahtanide qui décrivent ses exploits. Dans ces textes, Sanaa occupe une place importante et est évoquée comme étant le lieu de rassemblement de ses armées (M. Piotrovsky, 1984 : 129). La ville apparaît comme le point de départ de la conquête du monde ; les hauts faits d'As 'ad al-Kāmil, notamment la traversée de la mer des Ténèbres, étant quasiment identiques à ceux de Dū al-Qarnayn et à ceux du Roman d'Alexandre ${ }^{14}$.

En immergeant les terres entre Aden et la rive africaine de la mer Rouge, Dū al-Qarnayn délimite ainsi les contours de l'Arabie et dresse une muraille maritime devant les invasions venues d'Afrique. Ibn al-Muğāwir (1986 : 51) explicite cet exploit de Dū al-Qarnayn en rapportant que le Yémen était autrefois sous la coupe des Noirs ; le Sūdān, littéralement "Pays des Noirs", et l'Ethiopie semblant former dans son esprit une même entité. 
"Dans les temps anciens, la terre des Arabes et la terre du Soudan n'étaient pas séparées par la mer et le Yémen, aussi bien du temps de l'ignorance (ğăhiliyya) qu'à l'époque islamique, était occupé par le Soudan. Lorsque les flots montèrent, les Soudanais utilisèrent des bateaux pour se rendre sur l'autre rive et les îles appelées Mațārid al-hayl apparurent. Il est dit que les Arabes chassaient les chevaux au fond de cette mer lorsqu'elle s'asséchait. Ce lieu où poussaient l'herbe et les arbres est aussi dénommé Marābit al-ḩayl."

L'auteur (1986 : 107) cite le livre de Abū al-Tâami Ğayyāš ibn Nağğāḥ, al-Mufid fi ahbār Zabid, pour attester que

"la mer, à cet endroit, était autrefois un gué à eaux très basses qui permit aux Ethiopiens de venir conquérir la péninsule Arabique, de s'emparer de Sanaa et de s'avancer jusqu'à al-'Awāhil (le désert oriental du Yémen). Leur Etat qui chevaucha les périodes pré-islamique et islamique fut détruit par "Alī ibn Mahdi qui les annihila sans pitié en 554/1159-1160."

Les Ethiopiens d'Abraha et ceux des Banū Nağāḥ, les uns venus en 525, les autres, ayant fondé leur dynastie à Zabid au $\mathrm{XI}^{\mathrm{e}}$ siècle, sont perçus dans un même continuum temporel malgré les siècles qui les séparent.

Sur la terre d'Aden, Šaddād fait construire une porte et une prison, nouvelle limite circonscrivant le site ${ }^{15}$. A l'instar de Sem, il est en quête du lieu le plus propice et, en ce sens, l'élection d'Aden semble conférer à cette ville une stature inégalée. L'origine mythique de cette cité ne possède pourtant pas la même épure que celle de Sanaa. La volonté de Šaddād de la borner par une porte et de l'approvisionner en eau est contrariée par une force supérieure. Un des ğinn (génies) mettra soixante dix ans à construire la porte et l'autre n'achèvera jamais de creuser la rivière. Il est dans la nature du mythe d'être caractérisée par l'ambivalence. Ainsi, dans une autre partie du récit, les puits creusés à Lahğ fournissent l'eau potable aux habitants d'Aden. De fait, il se manifeste ici un semblant de contradiction qui reflète le caractère ambigu, sinueux, d'un acte de fondation qui ne laisse découvrir de la ville que l'image d'une porte et d'une prison. A contrario, dans les récits relatifs à Sanaa, le palais Gumdān, des jardins gigantesques ou des demeures innombrables, sinon le premier puits de la Création, produisent une image tangible de la ville. La topographie du site n'est pas un obstacle ou ne sert pas qu'à borner l'horizon. La montagne Nuqum est un point culminant à partir duquel Sem embrasse un univers non-clos ${ }^{16}$. De fait, dans une variante du mythe, la ville s'étend sur un territoire immense qui ne s'arrête qu'à Bașra. En outre, si l'on reprend en considération la figure héroïque d'As'ad al-Kāmil et la symbolique de puissance que représente le palais Ġumdān, Sanaa impose l'image d'une cité conquérante dont les armées atteindront les confins de la terre habitée.

\section{Epilogue}

Les origines mythiques de Sanaa et d'Aden rassemblent une pléiade de personnages coraniques qui attestent de l'importance symbolique de ces deux villes dans l'imaginaire arabe. Elles dévoilent de fait un ancrage yéménite de l'hiérohistoire musulmane qui est lié à l'élaboration d'une "épopée qahtanide" censée établir l'antériorité prophétique des Arabes du Sud ; As'ad al-Kāmil, entre autres exemples, est souvent présenté comme un monothéiste converti au christianisme qui aurait été le premier à recouvrir la Kaaba d'un voile (Ibn Hišăm, s.d. : 19-21 et Ibn al-Dayba', 1992 : 166-168). Cette épopée en arrive même à faire de Sanaa le berceau des Arabes si l'on se rapporte au texte d'Ibn al-Dayba'. Dans cette configuration mythique, Aden cristallise toutes les ambiguïtés du temps de la ğāhiliyya comme si sa situation de frontière, entre terre et mer, celle-ci séparant les Arabes des Africains, permettait de convoquer les dénégateurs, Qābīl et Šaddād, et le héros civilisateur, Dū al-Qarnayn, et de représenter ainsi la lutte du bien et du mal dans toutes ses incidences, métaphysiques et raciales. Polarisant un spectre très large de l'univers mythique yéménite, les récits de fondation de ces deux villes manifestent une complémentarité symbolique qui les a réunies dans le temps du mythe bien avant que l'histoire ne scellât leur union. 


\section{$138 /$ Franck Mermier}

NOTES

1. Grunebaum (G. E. von) $(1962: 31)$, introduit ainsi la question de la sacralité des cités islamiques: "Once safely and solidly Muslim, a city might wish to find itself a truly Islamic genealogy, so to speak, to convince itself that its origins or perhaps merely the origin of its more prominent sanctuaries, had been connected with some figure of Muslim mythology, preferably one of the prophets whom Muhammad had acknowledged as a predecessor".

2. “... Un mythe est donc aussi ce à quoi l'on croit. Toutefois, seul celui qui n'y croit pas peut soutenir qu' un autre y croit, et c'est en somme pour lui seul qu'il y a mythe ; pour l'autre le prétendu mythe n'en est pas un : qui irait affirmer qu'il accepte un mythe ? Autrement dit, un mythe en est un seulement pour celui qui le dénonce, non pour celui qui l'énonce, précisément parce que l'un et l'autre s'accordent à donner du mythe une définition négative : récit fabuleux, pensée confuse, idéalisation mensongère...", J. Pouillon (1980 : 85).

3. Il est cependant devenu rituel, pour Sanaa, d'invoquer le nom de son fondateur mythique, Sem, fils de Noé. A ce propos, voir, entre autres, le discours prononcé dans cette ville, le 19 décembre 1984, par M. Mahtar M'Bow, ancien directeur général de l'UNESCO, pour le lancement de la campagne internationale pour la sauvegarde de la vieille ville de Sanaa.

4. Texte tiré d'al-Iklil 8, éd. Muhammad al-Akwa', Damas : Mațba'at al-kātib al-'arabī, 1979, 34-36. Le même récit, quelque peu retouché, se trouve dans Sifat Ğazirat al-'arab, pp. 351-352

5. Ibn al-Muğāwir, Ğamāl al-Din Abū al-Fatḥ Yusūf b. Ya 'qūb b. Muhammad al-Šaybānī al-Dimašqi, décédé en 690/1291, est l'auteur supposé du Ta'rih al-Mustabșir. Voir G. Rentz (1990 : 904-906).

6. Parasange : unité de mesure d'origine persane équivalent à 6200 mètres (NDLR).

7. Plus loin dans son récit, Ibn al-Muğāwir (1986: 181) fait référence à une autre source attribuant la fondation de Gumdān à une femme appelée al-Zabbā’a. Il affirme en outre que ce palais, aux dimensions gigantesques et dont les limites atteignaient Wādi Zạhr, fut détruit par 'Umar ibn al-Haț̣âb, le deuxième Calife. Il existe évidemment d'autres données historico-légendaires sur ce palais, voir O. Löfgren (1977 : 1121-1122), et R. B. Serjeant et R. Lewcock (1983 : 44). Les tubba , rois légendaires du Yémen pré-islamique, sont évoqués dans des sourates coraniques (sourate de La Fumée, XLIV, verset 37 et sourate Qāf, L, verset 14). Sur la signification du terme tubba ${ }^{\prime}$, voir A. F. L. Beeston (1986: 6-8).

8. Sur As'ad al-Kāmil, voir A. F. L Beeston (1986 : 9-15) et l'étude magistrale de M. Piotrovsky (1984) rédigée en russe et que nous avons consultée dans sa traduction arabe.

9. Pour Le Caire, voir Maqrizi (s.d : 200-201) et pour Alexandrie, voir F. de Polignac (1987 : 56).

10. Dans le Coran "Bi-Cornu" n'est pas un conquérant mais un serviteur d'Allah qui, par la voie céleste, peut se transporter d'Occident en Orient pour accomplir des œuvres bienfaisantes et convertir les peuples païens"'(R. Blachère, 1966 : 745).

11. "Le peuple de 'Ād habitait les Ahqâf, c'est-à-dire les dunes de sable : la tradition les situait dans le pays de Wabâr, domajne merveilleux des djinns et des lions, en Arabie méridionale. 'Ād était le fils ou le descendant de Noé ; il épousa mille femmes, et il eut cent quatre mille enfants. Son fils Chaddâd construisit Iram aux piliers" (Gaudefroy-Demonbynes, 1969: 399). Le Prophète des “Ād était Hũd, voir sourate XI du même nom, versets 50-60. Le récit de Yãquat est mentionné dans W. Montgomery Watt (1990: 1303).

12. Il prolonge aussi le récit de l'acte fondateur de Dū al-Qarnayn par la relation de l'origine de la mer des Persans (al-A 'äğim) qui date, selon lui, de l'occupation d'Aden par les rois de Perse. Ceux-ci craignant que la ville ne soit trop vulnérable du fait de l'existence d'une bande de terre la reliant au rivage, décidèrent de l'isoler en la submergeant. Devenu une île, Aden ne fut plus accessible que par bateaux ce qui rendait pénible le transport des hommes et des chameaux. On construisit alors un pont appelé al-Maksir et la mer alentours restera dénommée, jusqu'au jour de la Résurrection, buhayrat al-'A 'āğim (Abū Mahrama, $1991:$ 35).

13. Le récit coranique de la digue élevée par Dū al-Qarnayn contre les peuples de Gog et Magog est relaté dans la sourate XVIII, versets 92-99.

14. Sur le Roman d'Alexandre et son influence sur la tradition musulmane, voir : A. Abel (1955) et J. A. Decourdemanche (1882).

15. Abū Mahrama (1991 : 2), en évoquant les différentes étymologies d'Aden, cite un auteur, al-Māzarī, qui explique son nom par le fait que tubba' y emprisonnait les criminels, Aden ('Adan) venant de 'udün qui signifierait "séjour" ('iqäma).

16. Le Nuqum possèdait une valeur symbolique importante pour le pouvoir zaydite. Chaque année, le $18 \mathrm{du}$ dū al-hiğğa, les imams réunissaient au pied de cette montagne des hommes de tribus et des habitants de la ville pour recevoir leur serment d'allégeance lors de la cérémonie du nušũr. Voir F. Mermier, 1991 : 177-180. 


\section{BIBLIOGRAPHIE}

ABel (A.), 1955, Le Roman d'Alexandre, Légendaire médiéval, Bruxelles, Office de Publicité, (Coll. Lebègue et Nationale).

Abū Mahrama, Abū Muhammad 'Abd Allāh..., 1991, Ta'rịh tağr 'Adan ma 'a nuhba min tawārīh Ibn al-Muğāwir wa al-Ğanadī wa al-Ahdal, édité par Oscar Löfgren, Le Caire, Maktaba Madbūli, $2^{e}$ éd.

BEESTON (A. F. L.), 1986, "Hamdāni and the Tabābi 'ah", dans al-Hamdāni, a Great Yemeni Scholar, Studies on the Occasion of his Millenial Anniversary, édité par Yusuf Muhammad Abdallah, Sanaa, Sanaa University, pp. 5-15.

BLACHERE (R.), 1966, Histoire de la littérature arabe. Des origines à la fin du XVe siècle de J.-C., Paris, Librairie Adrien-Maisonneuve.

DECOURDEMANCHE (J. A.), 1882, "La légende d'Alexandre chez les Musulmans", Revue de l'Histoire des Religions, $\mathrm{n}^{\circ}$ 6, pp. 98-112.

Gaudefroy-Demonbynes, (M.), 1969, Mahomet, Paris, Albin Michel, coll. L'évolution de l'Humanité.

GrunEBaum (G. E. von), 1962, "The Sacred Character of Islamic Cities”, Dans Mélanges Taha Hussein, édité par Abd el Rahman Badawi, Le Caire, pp. 25-37.

Hasan al-Hamdānī,1983, Sifat Ğazirat al- 'arab, édité par Muhammad 'Alī al-Akwa', Sanaa, Markaz al-dirāsāt wa-l-buhūt al-yamanī, $3^{e}$ édition.

Ḥasan al-Hamdānī,1986, Al-Iklil 8, édité par Muḥammad 'Ali al-Akwa', Beyrouth, Dār al-tanwïr.

Ibn Dayba', 1992, Našr al-mahăsin al-yamāniyya fi hașā'iș al-Yaman wa nasab al-qahṭāniyya, Beyrouth, Dār al-fikr al-mu āạir, Damas, Dār al-fikr.

Ibn Hišām, al-sira al-nabawiyya, $1^{\text {er }}$ volume, Le Caire, al-Maktaba al-tawfiqiyya, s.d.

Ibn al-Muğāwir, 1986, Sifat bilād al-Yaman wa Makka wa ba 'ḍ al-Hiǧăa al-musammã ta' rĭh al-mustabṣir, édité par Oscar Löfgren, Beyrouth, Dār al-tanwir, $2^{e}$ éd.

Ibn Rusteh, 1955, Les atours précieux, traduction de Gaston Wiet, Le Caire, Société de géographie d'Egypte.

LÖFGREN (O.), 1977, “Ghumdān”, Encyclopédie de l'Islam, II, Leyden, Brill, Paris, Maisonneuve et Larose.

Al-Maqrīīi, al-ditaṭ al-maqrizizya, vol. 2, Beyrouth, Dār 'ihyā' al-'ulūm, s.d.

MONTGOMERY Watt (W.),1990, "Iram", Encyclopédie de l'Islam, III, Leyden, Brill, Paris, Maisonneuve et Larose.

MERMIER (F.), 1991, "Récit d'origine et rituel d'allégeance : le jour de Ghadîr Khumm et la cérémonie du nushûr au Yémen”, Peuples Méditerranéens, Mythes et récits d'origine, n. 56-57, juil.-déc., pp.177-180.

PoullLon (J.), 1980, "La fonction mythique", Le temps de la réflexion, $\mathrm{n}^{\circ}$ 1, pp. 83-98.

Al-Rāzī, 1986, Ta'rih madinat Şan 'â', édité par Husayn 'Abd Allāh al- 'Amri, Sanaa, s.e., $2^{2}$ éd.

RENTZ (G.), Encyclopédie de l'Islam, nouvelle édition, vol. 3, pp. 904-906.

PIOTROVSKY (M.), 1984, Malhama 'an al-malik al-himyari As'ad al-Kãmil, traduit du russe par Šāhir Ğamāl Āgā, Sanaa, Wizārat al-'i 'lām wa al-țaqāfa.

POLIGNAC (F. de), 1987, "L'imaginaire arabe et le mythe de la fondation légitime", ROMM, 46, pp. 55-62.

SERJEANT (R. B.) et LewCOCK (R.), 1983, "The Church (al-Qalīs) of San 'ā' and Ghumdān Castle", in R. B. Serjeant et R. Lewcock éd., Şan 'á' an Arabian Islamic City, London, World of Islam Festival Trust, pp. 44-49. 